\title{
Evaluation of an online malnutrition management education module for general practitioners: the ONSPres project
}

\author{
A.A. Geraghty ${ }^{1,2}$, P. Dominguez Castro ${ }^{1,2}$, C.M.E. Reynoldd ${ }^{1,2}$, L. McBean ${ }^{1,2}$, B. Clyne ${ }^{3}$ \\ G. Bury ${ }^{4}$, F. Bourke ${ }^{5}$, C. Bradley ${ }^{5}$, K. Finnigan ${ }^{6}$, S. Clarke ${ }^{6}$, C. Perrotta ${ }^{1}$, L. McCullagh ${ }^{7}$, \\ C. Murrin ${ }^{1,2}$, E.R. Gibney ${ }^{2,8}$, S. Kennelly ${ }^{9}$ and C.A Corish ${ }^{1,2}$ \\ ${ }^{1}$ School of Public Health, Physiotherapy and Sports Science, University College Dublin, Belfield, Dublin, Ireland, \\ ${ }^{2}$ UCD Institute of Food and Health, University College Dublin, Belfield, Dublin, Ireland, \\ ${ }^{3}$ HRB Centre for Primary Care Research, Department of General Practice, Royal College of Surgeons in Ireland, \\ Dublin, Ireland, \\ ${ }^{4}$ School of Medicine, University College Dublin, Belfield, Dublin, Ireland, \\ ${ }^{5}$ Royal College of Surgeons in Ireland, Dublin, Ireland, \\ ${ }^{6}$ Department of Pharmacology and Therapeutics, Trinity Centre for Health Sciences, St James's Hospital, Dublin, \\ Ireland, \\ ${ }^{7}$ National Centre for Pharmacoeconomics, St James's Hospital, Dublin, Ireland, \\ ${ }^{8}$ School of Agriculture and Food Science, University College Dublin, Belfield, Dublin, Ireland and \\ ${ }^{9}$ National Primary Care Division, Community Funded Schemes Service Improvement, Mountmellick Primary Care \\ Building, Laois, Ireland
}

Malnutrition is common, yet under-diagnosed, amongst community-dwelling older adults ${ }^{(1)}$. General practitioners (GPs) are generally the first healthcare point of contact for older adults; however, currently there is little nutrition education in medical training and GPs report a lack of confidence in diagnosing and treating malnutrition ${ }^{(2,3)}$. To address this, GP interviews were carried out to establish preferences for the delivery and content of a malnutrition education programme and an online education module was designed. Efficacy of the module in improving knowledge and practice was evaluated.

Thirty-one GPs and GP trainees participated. The module covered the following topics: 'malnutrition definition, prevalence and latest evidence', 'identifying malnutrition in clinical practice', 'food-first advice', 'reviewing malnutrition' and 'oral nutritional supplements'. Knowledge was measured using a multiple-choice questionnaire (MCQ). Practice was evaluated using patient case studies at baseline, immediately post-module completion, and 6-weeks post-module. Case studies involved calculations of weight loss, malnutrition risk scores, and approaches to treatment and follow-up, which were then reviewed by a clinical specialist dietitian for older adults. Evaluation forms assessing feedback from the module were also completed. Differences between assessment performance were investigated using paired t-tests.

MCQ scores increased significantly from baseline to immediately post-module $(+25 \%, \mathrm{P}<0.001)$, with the greatest improvement in the 'identifying malnutrition in clinical practice' topic $(+47 \%, \mathrm{P}<0.001)$. Eleven GPs completed the 6-week MCQ when scores remained significantly increased from baseline $(+14 \%, \mathrm{P}=0.005)$, with the largest increase remaining in the identifying malnutrition in clinical practice' topic $(+40 \%, \mathrm{P}<0.001)$. Seventeen GPs completed the case studies with $85 \%$ correctly calculating malnutrition risk scores at baseline, increasing to $94 \%$ post-module. Identification of appropriate approaches to malnutrition treatment improved by $33 \%$ after module completion. GP feedback about the module was positive.

This online education module was well-received by GPs and was successful in improving malnutrition knowledge and practice, with this improvement being retained in the short-term. Future development of nutrition online education tools that promote evidencebased practical learning may represent an effective way to improve nutrition care provided by GPs.

\section{Acknowledgements}

This project was funded by the Health Research Board (HRB) under a quality and patient safety funding stream (RCQPS-2017-4).

\section{References}

1. Leij-Halfwerk S, Verwijs MH, van Houdt S, et al. (2019) Maturitas, 126, 80-9.

2. Nowson C (2020) BMJ NPH 0, 1-3

3. Dominguez Castro P, Reynolds CM, Kennelly S, et al. (2020) Clin Nutr ESPEN, 36, 116-27 